\title{
Molecular analysis of the mitochondrial markers COI, 12S rDNA and 16S rDNA for six species of Iranian scorpions
}

\author{
Parisa Soltan-Alinejad ${ }^{1,2}$, Javad Rafinejad ${ }^{3}$, Farrokh Dabiri' ${ }^{2}$, Piero Onorati ${ }^{4}$, Olle Terenius ${ }^{5}$ \\ and Ali Reza Chavshin ${ }^{1,2^{*}}$ (B)
}

\begin{abstract}
Objectives: Annually, 1.2 million humans are stung by scorpions and severely affected by their venom. Some of the scorpion species of medical importance have a similar morphology to species with low toxicity. To establish diagnostic tools for surveying scorpions, the current study was conducted to generate three mitochondrial markers, Cytochrome Oxidase I (COI gene), $12 \mathrm{~S}$ rDNA and $16 \mathrm{~S}$ rDNA for six species of medically important Iranian scorpions: Androctonus crassicauda, Hottentotta saulcyi, Mesobuthus caucasicus, M. eupeus, Odontobuthus doriae, and Scorpio maurus.

Results: Phylogenetic analyses of the obtained sequences corroborated the morphological identification. For the first time, $12 \mathrm{~S}$ rDNA sequences are reported from Androctonus crassicauda, Hottentotta saulcyi, Mesobuthus caucasicus and M. eupeus and also the $16 \mathrm{~S}$ rDNA sequence from Hottentotta saulcyi. We conclude that the mitochondrial markers are useful for species determination among these medically important species of scorpions.
\end{abstract}

Keywords: mtDNA, Scorpionidae, Buthidae, Iran

\section{Introduction}

Scorpions comprise more than 1500 species of which fifty species are considered of medical importance [1]. Annually, 1.2 million people are stung by scorpions [2], and severely affected by their venom [3]. The geographical and climatic diversity in Iran provides suitable conditions for a substantial number of scorpion species; as many as 52 species have been reported from Iran, although this number is not definitive [4]. Of these, at least seven species are dangerous to humans: Androctonus crassicauda Olivier, 1807; Apistobuthus pterygocercus Finnegan, 1932; Hottentotta saulcyi Simon, 1880; Hottentotta schach Birula, 1905; Hemiscorpius lepturus Peters, 1861;

\footnotetext{
*Correspondence: chavshin@umsu.ac.ir; chavshin@gmail.com

${ }^{1}$ Social Determinants of Health, Research Center, Urmia University

of Medical Sciences, Urmia, Iran

Full list of author information is available at the end of the article
}

Mesobuthus eupeus Koch, 1839 and Odonthubuthus doriae Thorell, 1876 [4]. The difficulties in identifying species based on morphological techniques [5], as well as the low number of studies [4], are two of the reasons for the uncertainty regarding the number of scorpion species existing in Iran. Using molecular markers in addition to morphological methods could be useful for a more accurate assessment of species diversity in Iran. One example where potential misidentification of scorpion species could be of importance, regards H. saulcyi and Scorpio maurus. The two species are similar in appearance, but while the venom for $H$. saulcyi has an $\mathrm{LD}_{50}$ value of $0.73 \mathrm{mg} / \mathrm{kg}$ and is considered a risk for humans, $S$. maurus has a venom with an $\mathrm{LD}_{50}$ value of $9.37 \mathrm{mg} / \mathrm{kg}$ that is considered harmless [6].

Among available molecular markers, the mitochondrial markers have shown to be useful for both species identification and phylogenetic evaluation of scorpion species. 
For example, analysis of intraspecific divergence between Androctonus scorpions using three mitochondrial markers (the COI gene, 12S rDNA and 16S rDNA), showed that these markers could explain the deep divergence sub-clades between Androctonus amoreuxi, A. australis and $A$. mauretanicus that also reflect differences in their venom production [7]. Also, COI and 16S rDNA markers have been used for investigating the evolution of Mesobuthus gibbosus in the Northeastern Mediterranean [8].

The current study was carried out to determine the COI, $12 \mathrm{~S}$ rDNA and 16S rDNA sequences of six medically important Iranian scorpions and to evaluate the utility of these markers for identification in comparison to morphology.

\section{Main text}

\section{Material and methods} Taxon sampling

Six species of medical importance were collected: Androctonus crassicauda Olivier, 1807; Hottentotta saulcyi Simon, 1880; Mesobuthus caucasicus Nordmann, 1840; Mesobuthus eupeus Koch, 1839; Odontobuthus doriae Thorell, 1876 and Scorpio maurus Ehrenberg, 1828. The samples were collected from the West Azerbaijan Province (Northwestern Iran) and the Qom Province (Central Iran; Additional files 1, 2: Tables S1, S2). The scorpions were captured at night using ultraviolet light (wavelength $366.3 \mathrm{~nm}$ ), which causes maximum fluorescence of their epicuticles. All collected specimens were stored in $96 \%$ ethanol. Specimens were identified using the keys of Farzanpay [9] and Dehghani and Valaie [10]. From the identified samples, one sample from each species at each site was selected for molecular investigations (Additional files 1, 2: Table S1, S2) and one leg was then removed from each specimen for DNA extraction.

\section{DNA extraction. COI, 12S rDNA and 16S rDNA amplification}

Genomic DNA was extracted from each specimen's leg tissue using AccuPrep ${ }^{\circledR}$ Genomic DNA Extraction kit (Bioneer, South Korea). The primers LCO1490 5'-GGT CAACAAATCATAAAGATATTGG- $3^{\prime}$ and HCO$^{\prime} 198$ 5'-TAAACTTCAGGGTGACCAAAAAATCA-3' were used for amplification of the COI gene [11]. The primers Sc-12F $5^{\prime}$-AGAGTGACGGGCAATATGTG-3' and Sc-12R 5' - CAGCGGCTGCGGTTATAC-3' were used for amplification of $12 \mathrm{~S}$ rDNA [12]. The primers Sc-16F 5'-CGATTTGAACTCAGATCA-3' ${ }^{\prime}$ and Sc-16R $5^{\prime}$ - GTG CAAAGGTAGCATAAT- $3^{\prime}$ were used for amplification of $16 \mathrm{~S}$ rDNA [13]. PCR conditions for amplification of all fragments were as follows: initial denaturation at $94{ }^{\circ} \mathrm{C}$ for $5 \mathrm{~min}$; 30 cycles of $\left[94{ }^{\circ} \mathrm{C}\right.$ for $30 \mathrm{~s}, 48{ }^{\circ} \mathrm{C}$ for $30 \mathrm{~s}, 72{ }^{\circ} \mathrm{C}$ for $30 \mathrm{~s}$ ] and a final extension at $72{ }^{\circ} \mathrm{C}$ for $7 \mathrm{~min}$.

\section{Phylogenetic analysis}

All amplicons of the three fragments were sequenced and acquired consensus sequences were analyzed using BLAST (http://blast.ncbi.nlm.nih.gov/Blast.cgi) in order to find and include similar sequences in the phylogenetic analysis. The phylogenetic relationship of the scorpions in the current study was inferred by using the Maximum Likelihood tree building algorithm and the Tamura-Nei model [14]. Initial tree(s) for the heuristic search were obtained automatically by applying Neighbor-Join and BioNJ algorithms to a

(See figure on next page.)

Fig. 1 Morphological characteristics of the scorpion species of the current study. From the family Scorpionidae, which is characterized by a pentagonal sternum (a), is the only representative Scorpio maurus which is characterized by no-granule prosoma (b1) and a short and bulky pincer (b2). Species belonging to the family Buthidae have a triangular sternum as a diagnostic character (c). The Hottentotta saulcyi has a dark colored Prosoma (d1) and regular teeth of the abdominal region of the fifth segment of the post-abdominal and caudal vesicle (d2). The morphological characteristics of Androctonus crassicauda are the dark color of this species and the special tufts in the tail (e1) and, most importantly, the existence of three granules under the tooth of the fingers of the pincer (e2). Odontobuthus doriae has circular-end long teeth in the abdominal region of the

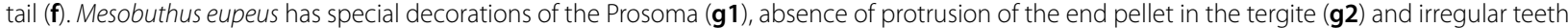
with a specific design in the abdominal region of the tail (g3). Mesobuthus caucasicus has granules along each other in carapace (h1), the complete protrusion of the end granule in the tergite region (h2), as well as the presence of an outer sub-granule being smaller than the inner sub-granule in the dental rows of the pincer ( $\mathbf{h} \mathbf{3})$ 


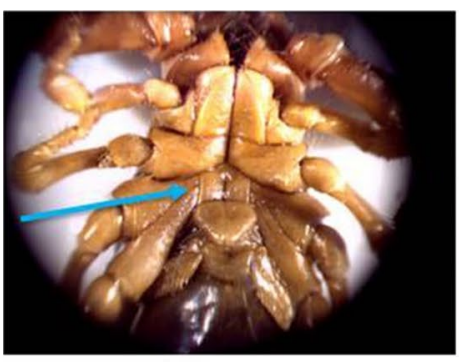

a

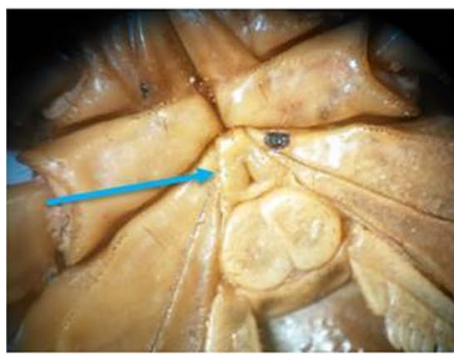

c

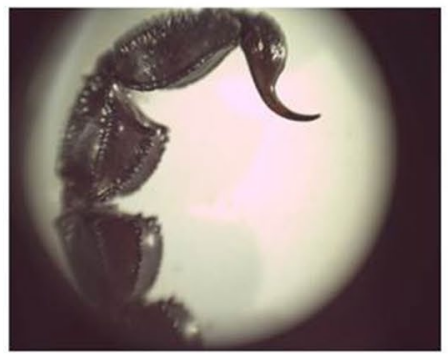

e1

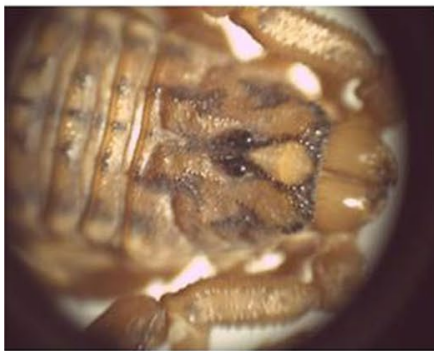

g1

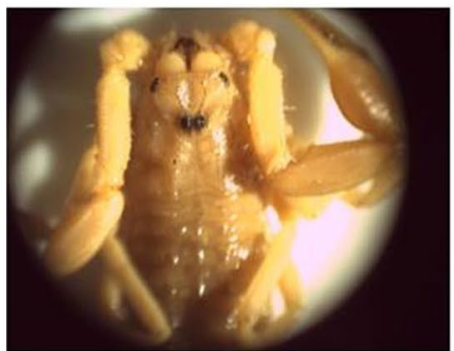

h1

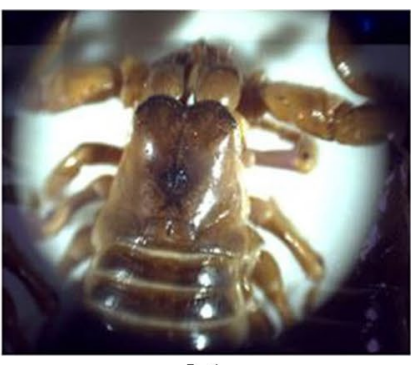

b1

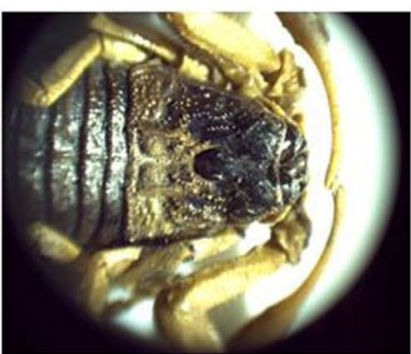

d1

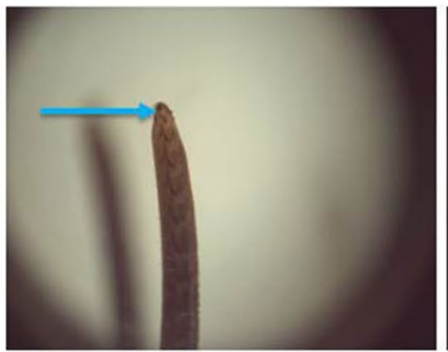

e2

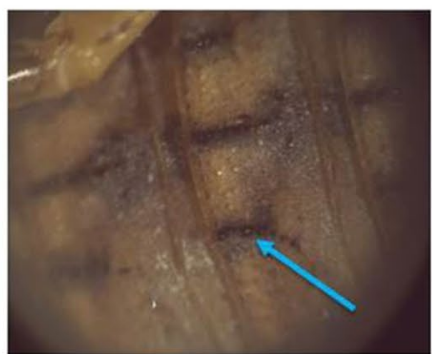

g2

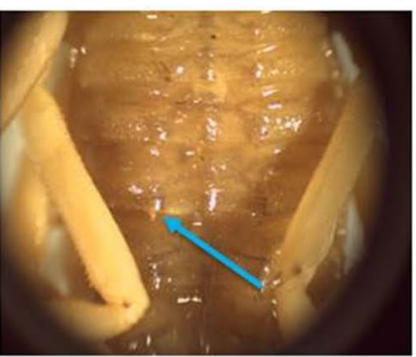

h2

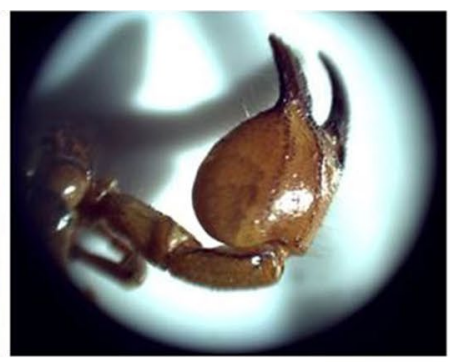

b2

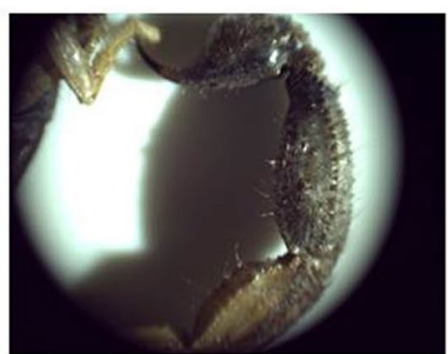

d2

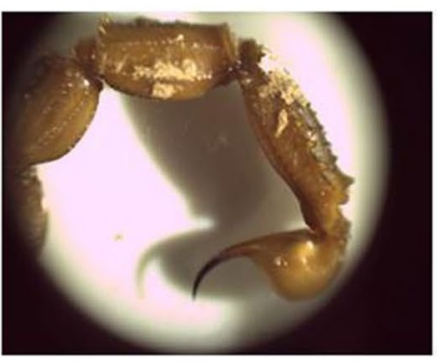

f

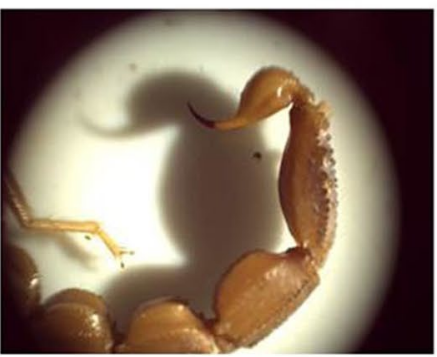

g3

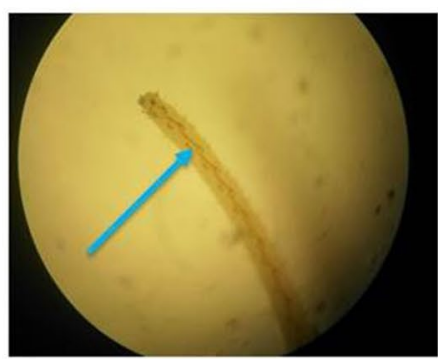

h3 
matrix of pairwise distances estimated using the Maximum Composite Likelihood (MCL) approach, and then selecting the topology with superior log likelihood value. Evolutionary analyses were conducted in MEGA X [15].

\section{Results and discussion}

In the current study, six species of Buthidae and Scorpionidae scorpions from northwestern and central Iran were identified based on morphological characteristics (Fig. 1). We amplified the COI gene, $12 \mathrm{~S}$ rDNA and $16 \mathrm{~S}$ rDNA and a representative subset was deposited in GenBank; accession numbers are shown in Additional files 1, 2: Table S1, S2 and in Fig. 2. The acquired sequences of $12 \mathrm{~S}$ rDNA fragments in the species Androctonus crassicauda, Hottentotta saulcyi, Mesobuthus caucasicus, $M$. eupeus as well as 16S rDNA in Hottentotta saulcyi are novel. We also consider our COI sequence of $H$. saulcyi to be the first for this species, since the existing COI sequence in GenBank (KU341989) seems to be from a misidentified specimen (Fig. 2a). Additionally, the sequences of 16S rDNA of A. crassicauda, M. caucasicus, and M. eupeus, the $12 \mathrm{~S}$ rDNA sequence of Odontobuthus doriae, and all three mitochondrial gene sequences of Scorpio maurus are the first reported from Iran.

In this study, we successfully used $16 \mathrm{~S}$ rDNA to differentiate between genera, and sequences clustered well on the species level (Fig. 2b). Likewise, Cytochrome Oxidase I (COI) and 16S rDNA fragments had the appropriate capability for the differentiation of four
Eurasian scorpion species (Mesobuthus caucasicus, $M$. cyprius, M. eupeus, and M. gibbosus; [16], which is in accordance with our results showing effective differentiation of Mesobuthus at the species level using all three mitochondrial markers (Fig. 2).

There has been taxonomic controversy over the placement of Mesobuthus caucasicus, which has been proposed to belong to the genus Olivierius [4, 9, 17-19] while other researchers prefer to maintain the original scientific name Mesobuthus caucasicus [16, 20-22]. Our phylogenies of all three mitochondrial markers strongly suggest that Mesobuthus caucasicus should indeed be included in the genus Mesobuthus (Fig. 2) and confirmed by placing Opisthacanthus asper as a rooted outgroup (Fig. 2).

In all, we have in this paper shown that COI, $12 \mathrm{~S}$ rDNA and 16S rDNA are efficient markers for phylogenetic discrimination of these Iranian scorpions and set the foundations for the correct identification of these medically important species.

\section{Limitations}

Due to the fact that the samples analyzed in this analysis were collected from limited areas, the collection of samples and species from wider areas could better indicate potential differences in morphological characteristics and genetic markers. Also, the selection of one sample of any scorpion species in each location for molecular investigation, as a limiting factor in the present study, should be considered.

(See figure on next page.)

Fig. 2 Molecular phylogenetic analysis of a COl, using the Maximum Likelihood method with 1000 bootstrap replications. The tree with the highest log likelihood (-4375.20) is shown. There were a total of 698 positions in the final dataset. The percentage of trees in which the associated taxa clustered together is shown next to the branches. The tree is drawn to scale, with branch lengths measured in the number of substitutions per site. Asterisks indicate sequences obtained in this project. b $16 \mathrm{~S}$ rDNA, using the Maximum Likelihood method with 1000 bootstrap replications. The tree with the highest log likelihood (-3635.89) is shown. There were a total of 489 positions in the final dataset. Asterisks indicate sequences obtained in this project. c $12 \mathrm{~S}$ rDNA, using the Maximum Likelihood method with 1000 bootstrap replications. The tree with the highest log likelihood

$(-4080.84)$ is shown. There were a total of 524 positions in the final dataset. Asterisks indicate sequences obtained in this project 
a

70 [ MH352610 Androctonus crassicauda Iran Zanjan

99 MK814933 Androctonus crassicauda I ran Makoo*

\begin{tabular}{|} 
MK814934 Androctonus crassicauda Iran Sardasht* \\
HM567333 Androctonus crassicauda Iran Khorasan
\end{tabular}

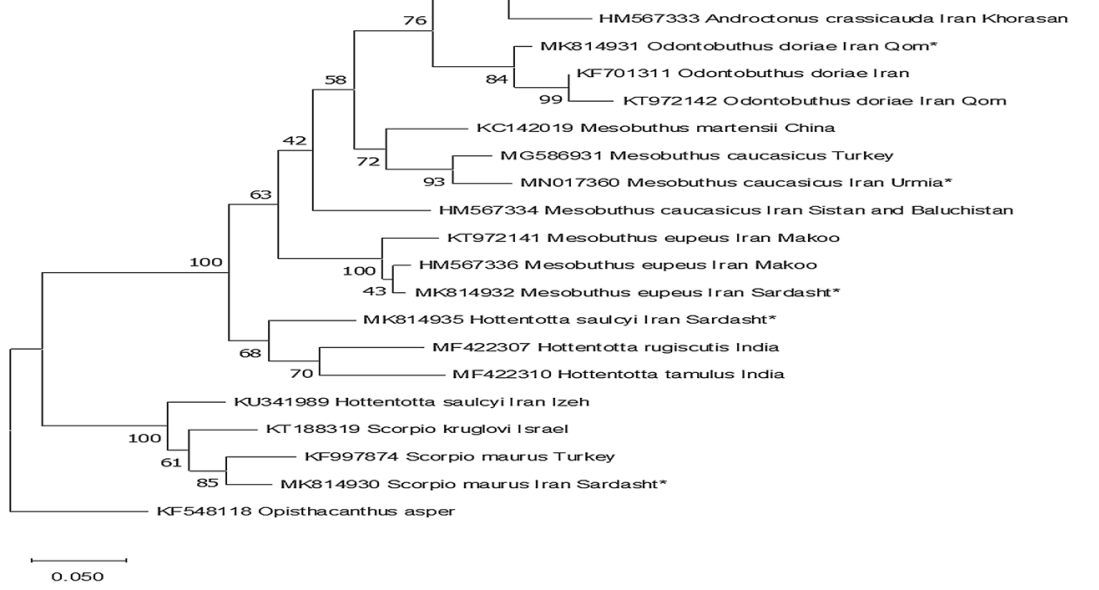

b

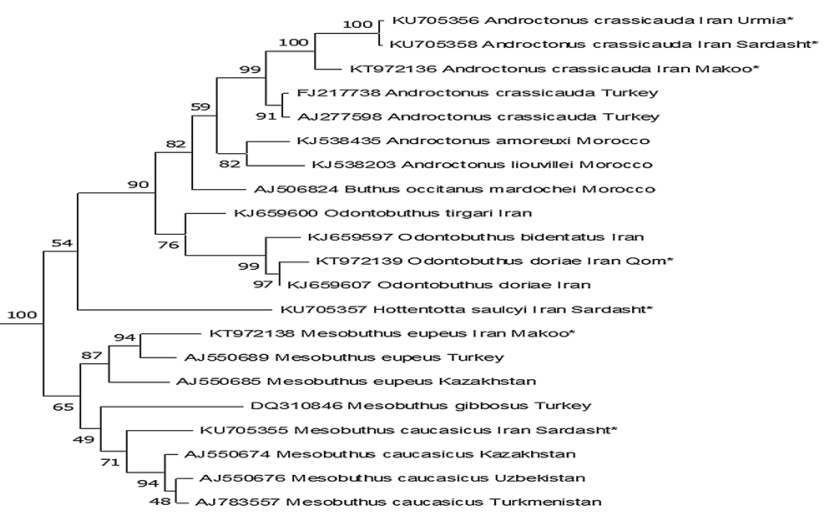

KU705354 Scorpio maurus Iran Mahabad

90 KT 188178 scorpio tuscus Israel

61 KT188203 Scorplo kruglovi I srae

$\stackrel{0.050}{0}$

c

98 [ KU705366 Androctonus crassicauda Iran Urmia*

100 KU705367 Androctonus crassicauda Iran Makoo*

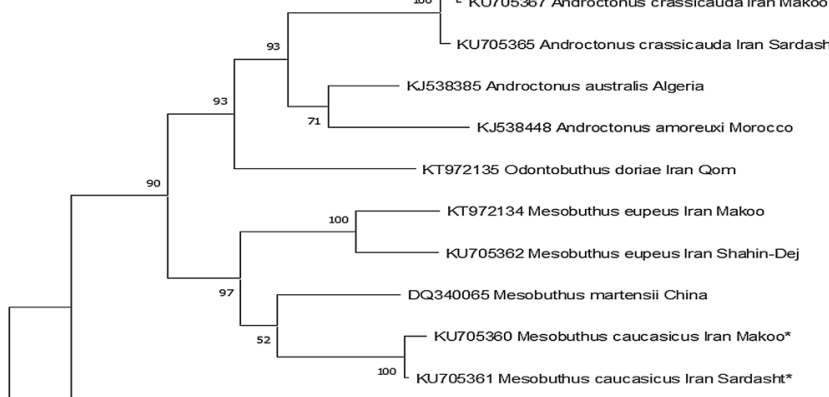

KU705364 Hottentotta saulcyi Iran Sardasht

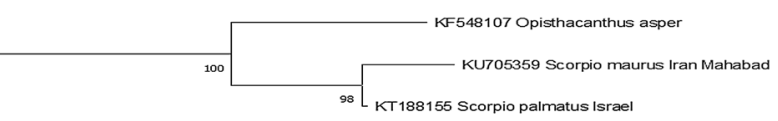




\section{Supplementary Information}

The online version contains supplementary material available at https://doi. org/10.1186/s13104-021-05449-3.

Additional file 1: Table S1. Summary of scorpion taxa collected, sampling localities and accession numbers of acquired $12 \mathrm{~S}$ and $16 \mathrm{~S}$ sequences.

Additional file 2: Table S2. Summary of scorpion taxa collected, sampling localities and accession numbers of acquired $\mathrm{COI}$ sequences.

\section{Abbreviations}

COI: Cytochrome Oxidase 1; 12S rDNA: 12S ribosomal DNA; 165 rDNA: 165 ribosomal DNA.

\section{Acknowledgments}

This article is part of the results of the first author's dissertation for fulfillment of MSc degree in Medical Entomology and Vector Control from the Department of Medical Entomology and Vector Control, School of Public Health, Urmia University of Medical Sciences, Urmia, Iran. This study was supported by the Urmia University of Medical Sciences (UMSU), Urmia, Iran (Project no. 34-1644).

\section{Authors' contributions}

ARC designed and supervised the study. PSA, JR, FD and PO did the field and laboratory activities. ARC and OT analyzed the Data. Also, ARC and OT wrote the draft and finalized the Draft. All authors read and approved the final manuscript.

\section{Funding}

This study was financially supported by the Urmia University of Medical Sciences (UMSU), Urmia, Iran (Project no. 34-1644). The funder had no role in data collection, analysis, interpretation and presentation.

\section{Availability of data and materials}

The datasets used and/or analyzed during the current study are available from the corresponding author on reasonable request.

\section{Ethics approval and consent to participate}

This project was approved by the Ethics Committee of Urmia University of Medical Sciences.

\section{Consent for publication}

Not applicable.

\section{Competing interests}

The authors declare that they have no competing interests.

\section{Author details}

${ }^{1}$ Social Determinants of Health, Research Center, Urmia University of Medical Sciences, Urmia, Iran. ${ }^{2}$ Department of Medical Entomology and Vector Control, School of Public Health, Urmia University of Medical Sciences, Urmia, Iran. ${ }^{3}$ Department of Medical Entomology and Vector Control, School of Public Health, Tehran University of Medical Sciences, Tehran, Iran. ${ }^{4}$ Department of Ecology, Swedish University of Agricultural Sciences, Uppsala, Sweden. ${ }^{5}$ Department of Cell and Molecular Biology, Uppsala University, Uppsala, Sweden.

Received: 15 November 2020 Accepted: 12 January 2021 Published online: 01 February 2021
References

1. Stockmann RY, Flay E, Nicholas coaut tr Roland Stockmann EY: Scorpions of the World; 2010

2. Chippaux J-P, Goyffon M. Epidemiology of scorpionism: a global appraisal Acta Trop. 2008;107(2):71-9.

3. Angsanakul J, Sitprija V. Scorpion venoms, kidney and potassium. Toxicon. 2013;73:81-7

4. Dehghani $R$, Fathi B. Scorpion sting in Iran: a review. Toxicon. 2012:60(5):919-33.

5. Lourenço WR. Why does the number of dangerous species of scorpions increase? The particular case of the genus Leiurus Ehrenberg (Buthidae) in Africa. J Venom Anim Toxins incl Trop Dis. 2020;26.

6. Yağmur EA, Özkan Ö, Karaer KZ. Determination of the median lethal dose and electrophoretic pattern of Hottentotta saulcyi (Scorpiones, Buthidae) scorpion venom. J Arthropod Borne Dis. 2015;9(2):238.

7. Coelho P, Sousa P, Harris D, van der Meijden AJAT. Deep intraspecific divergences in the medically relevant fat-tailed scorpions (Androctonus, Scorpiones). Acta Tropica. 2014;134:43-51.

8. Parmakelis A, Stathi I, Chatzaki M, Simaiakis S, Spanos L, Louis C, Mylonas M. Evolution of Mesobuthus gibbosus (Brullé, 1832)(Scorpiones: Buthidae) in the northeastern Mediterranean region. Mol Ecol. 2006;15(10):2883-94.

9. Farzanpay R. Knowing scorpions. Tehran: University Publication; 1987.

10. Dehghani R, Valaie N. The review of classification of scorpions and their diagnostic key of Iran scorpions. Feyz J. 2004;8(32):62-84.

11. Folmer $\mathrm{O}$, Black $M$, Hoeh W, Lutz R, Vrijenhoek R. DNA primers for amplification of mitochondrial cytochrome c oxidase subunit I from diverse metazoan invertebrates. Mol Mar Biol Biotech. 1994;3(5):294-9.

12. Pedroso DDOG. Phylogeography of the scorpion genus Buthus in the Maghreb region. Universidade do Porto 2012.

13. Gantenbein $B$, Fet $V$, Largiadèr $C$, Scholl A. First DNA phylogeny of Euscorpius Thorell, 1876 (Scorpiones, Euscorpiidae) and its bearing on taxonomy and biogeography of this genus. Biogeographica (Paris). 1999;75(2):49-65.

14. Tamura K, Nei M. Estimation of the number of nucleotide substitutions in the control region of mitochondrial DNA in humans and chimpanzees. Mol Biol Evol. 1993;10(3):512-26.

15. Kumar S, Stecher G, Li M, Knyaz C, Tamura K. MEGA X: molecular evolutionary genetics analysis across computing platforms. Mol Biol Evol. 2018;35(6):1547-9.

16. Gantenbein B, Fet $V$, Gromov AV. The first DNA phylogeny of four species of Mesobuthus (Scorpiones, Buthidae) from Eurasia. J Arachnol. 2003;31(3):412-20.

17. Cao Z, Di Z, Wu Y, Li W. Overview of scorpion species from China and their toxins. Toxins. 2014;6(3):796-815.

18. Dehghani R, Djadid ND, Shahbazzadeh D, Bigdelli S. Introducing Compsobuthus matthiesseni (Birula, 1905) scorpion as one of the major stinging scorpions in Khuzestan, Iran. Toxicon. 2009;54(3):272-5.

19. Vatani $H$, Khoobdel M. Scorpion fauna in Taybad region and scorpion sting status in military environment. J Mil Med. 2009;11(1):7-11.

20. Fet V. Fauna and zoogeography of scorpions (Arachnida: Scorpions) in Turkmenistan. Berlin: Biogeography and ecology of Turkmenistan. Springer; 1994. p. 525-34.

21. Jalali A, Rahim F. Epidemiological review of scorpion envenomation in Iran. Iran J Pharm Res. 2014;13(3):743-56.

22. Karataş A. Mesobuthus caucasicus (Nordmann, 1840)(Scorpiones: Buthidae) in Turkey. Euscorpius. 2015;2005(25):1-7.

\section{Publisher's Note}

Springer Nature remains neutral with regard to jurisdictional claims in published maps and institutional affiliations. 